\title{
TUNNELING IONIZATION BUNCH LENGTH MONITOR FOR THE ULTRARELATIVISTIC COMPRESSED ELECTRON BEAMS
}

\author{
A. Murokh, UCLA, CA 90095-1547, USA
}

\begin{abstract}
The electric field intensity of the compressed ultrarelativistic electron beams is approaching $\mathrm{GV} / \mathrm{m}$ levels, which is sufficient to cause observable tunneling effect in the low band gap materials. In this article the tunneling ionization rate is estimated for the experimentally available electron beam parameters, and a proposed proof of principle experiment is outlined. Tunneling effect has exponential dependence on the electric field strength; thus being very sensitive to the electron beam peak current. This non-linear dependence opens up a possibility to construct inexpensive, single shot and non-destructive peak current diagnostics for the ultrarelativistic compressed electron beams.
\end{abstract}

\section{INTRODUCTION}

As the advanced photo-injector facilities around the world utilize bunch compression techniques to enhance brightness in the beams [1-2], the ability to measure peak currents of such beams become critical to the successful facility operations. The techniques employed for such measurements include deflecting cavities [3], streak cameras, CTR monitors (Coherent Transition/Diffraction Radiation) [4] and electro-optical systems [5]. All these techniques have been used in the past, yet they have significant limitations. Deflecting cavities are destructive to the beam, streak cameras do not have sufficient resolution for sub-picosecond beams, CTR measurements are time consuming and the data analysis requires making assumptions about the beam shape [6], and electro-optical techniques require rather sophisticated experimental apparatus.

In the same time, for the advanced facilities, such as LCLS (Linear Coherent Light Source), accurate peak current measurements are not only essential for the proper characterization of the beam, but could also provide a unique opportunity to serve as a feedback to compensate for the fast drifts in the RF phase of the injector/linacs [7]. Indeed, the peak current of the electron beam, once it is subject to bunch compression in the chicane, become most sensitive to the minor variation in the RF phase. The ability to make single shot, non-destructive, direct measurements of the peak current in a simple and easily interpretable way would be of the great value as both diagnostic technique and feedback mechanism.

Even the fastest electronic devices such as ICT or stripline monitors rely on the processes of much longer timescale than a time structure of the picosecond electron beams which are subject of the interest. As a result the measurements become integrated over the bunch length and not sensitive to the longitudinal profile of the beam. One physical process, however, which has much shorter characteristic time is quantum tunneling, which takes place when the dielectric material is exposed to the intense electric field. The tunneling process has a time scale of the order $t \sim h / k T$ (which is about 100 femtoseconds for $T \sim 77 \mathrm{~K}$ ). Most importantly, the rate of quantum tunneling is exteremly sensitive to the peak field, rather than the time-integrated radiation intensity flux. Hence, if one would observe the tunnneling process in the material sample placed near the path of the ultrarelativistic, high current electron beam, the rate of the process will be most sensitive to the peak current in the beam, rather than the integrated charge in the bunch.

This feature makes potential quantum tunneling device an attractive alternative for the fast electron beam diagnostics. In this paper it is proposed to use quantum tunneling effect induced in the doped semiconductor materials by the bulk electric field of the ultrarelativistic compressed electron beam as a single-shot nondestructive peak current monitor.

\section{STATIC TUNNELING MODEL}

Tunneling related effects induced by the powerful laser beams have been thoroughly studied both theoretically and experimentally in the recent years [8]. The static probability of direct tunneling in the electric field has exponential dependence on the field strength [9],

$$
w(t)=4 \square o=\left.\frac{E_{i}}{E_{h}}\right|^{5 / 2} \frac{E_{a}}{E(t)} e^{\square \frac{2}{3}\left[\frac{E i}{E h} \theta^{3 / 2} \frac{E a}{E(t)}\right.}
$$

where $\square_{0}=2 \square \square \square^{2} m_{\mathrm{e}} c^{2} / h$ is the atomic frequency unit, $E_{\mathrm{i}}$ and $E_{\mathrm{h}}$ are the ionization potentials of the sample material and hydrogen respectively, $E(t)$ is time-dependent electric field strength, and $E_{\mathrm{a}}=e / 4 \square\left[r_{\mathrm{b}}{ }^{2}\right.$ is an atomic unit of the electric field. In the laser induced tunneling experiments the observed ionization rate become frerquency dependent below far infrared wavelength of the applied radiation. Interpolating these findings towards the electron beam induced tunneling, one would obtain the limit where static formalism applies [10],

$$
E_{i}<U \square \frac{e^{2} E^{2}}{4 m_{e}} \square \square^{2}
$$

where $\square_{\square}$ is the bunch length. 
This condition may not be satisfied, for instance in the case of the low charge bunches, or if the electron beam field is microbunched at the higher frequencies. In that case, following the analogy with the theory of the laser induced tunneling, the process deviates from the static limit (1) and become frequency dependent, being dominated by phonon-assisted ionization in solids [10], or multi-photon absorption in gases [11]. For the typical high brightness electron beam of the interest here, the condition given in (2) is satisfied; hence, below only the static tunneling ionization model will be considered. Clearly, more detailed analysis in the future should consider all the relevant processes.

Using the static tunneling model, one can calculate the physically achievable electron beam parameters, where the effect can be observed. For a typical high brightness ultrarelativistic electron beam $([>100)$ the electric field temporal profile mirrors the shape of the electron beam longitudinal distribution, as long as it is sampled sufficiently close to the beam path:

$$
E(r, t) \square \frac{I(t)}{2 \square \square r c}
$$

Given post-compression electron beam peak currents values of the order $I_{\text {peak }} \sim 1 \mathrm{KA}$, the field intensity reaches up to $100 \mathrm{MV} / \mathrm{m}$, as far as few millimiters away from the beam path.

Such field intensities are generally not sufficient to generate observable tunneling ionization in the dielectrics at the room temperature (which typically have a band gap of around $10 \mathrm{eV}$ ). However, if one would use low band gap doped semiconductor materials the field of that amplitude can generate an observable tunneling ionization. Of course, the target material should be cooled under liquid nitrogen, so that the free carriers generation could be dominated by quantum tunneling effect and not the thermal noise. For instance, in doped germanium (which was used in the measurements shown

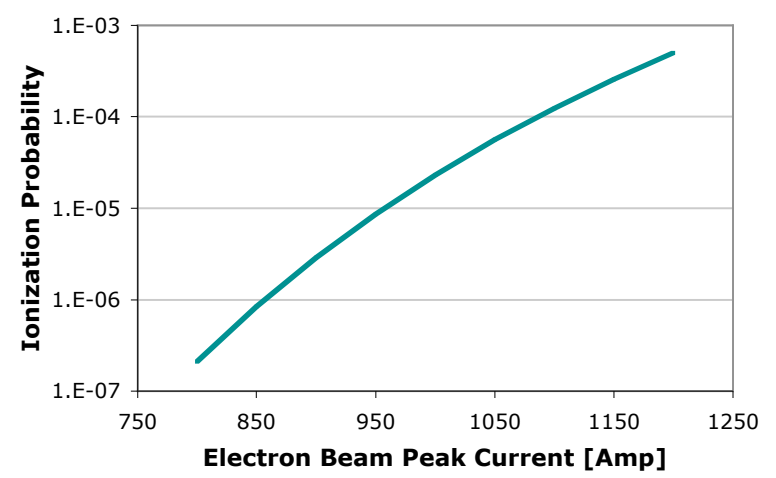

Figure 1: Static tunneling probability for the gold impurities in germanium target placed $3 \mathrm{~mm}$ away from the path of $1 \mathrm{nC}$ ultrarelativistic electron beam.

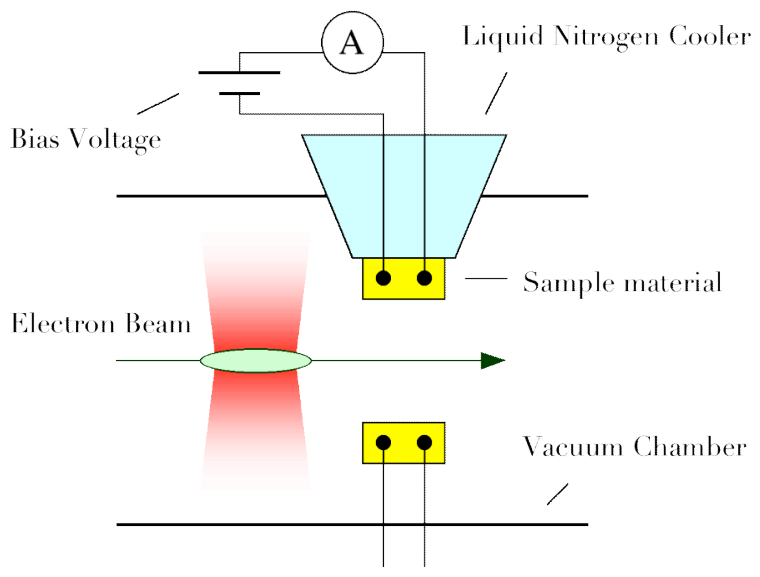

Figure 2: Experimental apparatus to measure tunneling effect in the field of the electron beam. Nitrogen cooled semiconductor sample is placed near the beam path with the bias voltage applied. Current induced by the beam

passage would be mostly due to the tunneling at the dopant sites.

in [10]), the ionization energy for deep impurities is of the order of $E_{\mathrm{i}} \sim 100 \mathrm{meV}$. In such materials, deep impurities tunneling ionization has been extensively studied in the fields of the $\mathrm{THz}$ laser beams [12], which intensities were similar or smaller than the field values in quest.

As an example, one can consider cooled Ge:Au sample $\left(E_{\mathrm{i}} \sim 150 \mathrm{meV}\right)$, and $1 \mathrm{nC}$ electron beam passing $3 \mathrm{~mm}$ away from the target. The ionization rate calculated through time integration of the equation (1) will exhibit near exponential dependence on the electron beam peak current (Figure 1).

\section{EXPERIMENTAL CHALLENGES}

To conduct a proof of principle experiment, one has to cool the sample material down to the liquid nitrogen temperature, in order to minimize the thermal noise (Figure 2). When bias voltage (usually of the order of 5 $\mathrm{V})$ is applied, and electron beam is not present, there should be no current in the circuit. However, when a high peak current electron beam passes near the biased sample, tunneling ionization at impurities sites "opens up" the circuit and the charge flow can be measured. In that case the integrated charge flux through the circuit should be a very non-linear function of the electron beam peak current. Following the model shown in Figure 1, a $10 \%$ variation in the electron beam peak current should lead to the order of magnitude change in the measured signal area. Having such device in the beamline, would enable to observe non-destructively very small changes in the electron beam peak current and compensate for them if needed. Proper calibration against some other diagnostics may even allow the absolute measurements.

As the electron beam field strength decays radialy, two identical samples should be placed symmetrically around 
the path of the beam. That would allow reducing measurement errors associated with the beam position jitter. In fact, to remove the errors associated with the jitter in both planes one can consider the 4 samples system, which can also act like a BPM (Beam Position Monitor), similar to the stripline.

To improve signal to noise ratio, it is critical to prevent the target material from being irradiated by the shorter wavelength sources. Particularly problematic could be IR, optical and X-ray radiation produced by the electron beam itself upstream along the beam line. Good shielding material to reduce such problem would be silicon, which is opaque for the optical wavelengths, and yet have relatively low index of refraction for the electron beam induced field.

Another important consideration is secondary ions produced in the host material. The tunneled electrons can be accelerated by the electron beam field and reach energies above the ionization potential of the host material. Such electrons would ionize host atoms, increasing the overall ions population, and such process can generate a correction to the behaviour presented in the Figure 1; an effect somewhat similar to the one postulated in [13]. This additional ionization enhancement should be an important factor to consider, while optimizing the distance from the electron beam path to the sample for the specific electron beam parameters.

\section{CONCLUSION}

The concept of the electron beam bulk field induced quantum tunneling, introduced in this paper, can probably be expanded to a much deeper level of theoretical understanding, which at present is way beyound the author's horizon. Yet one can find it rather meaningful to conduct a pilot experiment and, if successful, use it as a reference point for further theoretical investigation of the tunneling phenomena. Such effort would not only be valuable as an electron beam diagnostics research, but also of the general interest to the material science community.

\section{ACKNOWLEDGEMENT}

The work presented in this paper was impacted by helpful discussions with C. Pellegrini, P. Krejcik, G. Travish and S. Ganichev, and also supported by the ONR Grant \#N00014-02-1-0911.

\section{REFERENCES}

[1] M. Cornacchia et. al., A Sub-Picosecond Photon Pulse Facility for SLAC, SLAC-Pub-8950, (2001).

[2] L. Serafini and M. Ferrario, in Physics of, and Science with, the X-ray Free-Electron Laser, edited by S. Chattopadyay et al., AIP Conf. Proc. No. 581 (AIP, New York), 87, (2001).

[3] R.H. Miller, R.F. Koontz, D.D. Tsang, The SLAC Injector, IEEE Trans. Nucl. Sci., 804, (1965).

[4] U. Happek and A. J. Sievers, Phys. Rev. Lett. 67, 2962 (1991).

[5] I. Wilke et al., Phys. Rev. Lett 88, 124801, (2002).

[6] A. Murokh et al., Nucl. Instr. and Meth. A 410, 452 (1998).

[7] P. Emma, private communication.

[8] S.D. Ganichev et al., Phys. Rev. Lett. 80, 2409, (1998).

[9] P.B. Corkum, N.H. Burnett and F. Brunel, Phys. Rev. Lett. 62, 1259, (1989).

[10] S.D. Ganichev, W. Prettl and P.G. Huggard, Phys. Rev. Lett. 71, 3882, (1998).

[11] S. Augst, et al., Phys. Rev. Lett. 63, 2212, (1989).

[12] S.D. Ganichev et al., Phys. Rev. Lett. 75, 1590, (1995).

[13] A. Murokh et. al., Limitations on Resolution of YAG:Ce Beam Profile Monitor for High Brightness Electron Beam, Proceeds. 2nd ICFA Advanced Accelerator Workshop, edited by J. Rosenzweig and L. Serafini, (Los Angeles, 1999), World Scientific, $564,(2000)$. 